\title{
DESIGN OF STATIC WDM MESH NETWORKS WITH DEDICATED PATH-PROTECTION
}

\author{
Simone De Patre ${ }^{1}$, Guido Maier ${ }^{1}$, Mario Martinelli ${ }^{1 *}$, and Achille Pattavina ${ }^{2}$ \\ ${ }^{1}$ CoreCom, \\ Via Ampere, 30 - 20131 Milan, Italy \\ \{maier, depatre, martinelli\}@ corecom.it \\ ${ }^{2}$ Dept. of Electronics and Information, Politecnico di Milano, \\ P.za Leonardo da Vinci, 32 - 20133 Milan, Italy \\ pattavina@elet.polimi.it
}

Abstract Failure-protection of Wavelength Division Multiplexing networks is a hot researchtopic today. In this paper we focus on optical path protection in multifiber mesh networks. We present a heuristic method to assign working and protection capacity to a set of static optical-connection requests under the path-disjoint constraint. Routing, fiber and wavelength assignment are jointly performed for all the lightpaths with the aim of minimizing the number of fibers in the network. The proposed heuristic approach allows to obtain good suboptimal results compared to the exact integer-linear-programming optimization. We discuss the optimization of particular case-study networks in order to analyze the influence of different link-disjoint-path search algorithms on the final results and of various design criteria.

Keywords: Wavelength Division Multiplexing, optical network design, WDM path-protection, heuristic methods

\section{Introduction}

The issue of survivability of optical connections has become of outstanding importance today for the WDM world. This is due to the obvious reason that a loss of a high-speed optical connection operating at bit-rates of $10 \mathrm{Gbit} / \mathrm{s}$ or higher, even for few seconds, means a huge waste of data. Electronic transport layers (SDH/Sonet and $\mathbb{I P}$ ) already offer a full range of well known and widespread failure recovery mechanisms: at this layers, however, network reac- 
tions are relatively slow and quite complex to manage, due to the large amount of signaling and control information and to difficulties in interoperating with the optical layers. For this reason the research community, standardization organizations [1] and a large number of system vendors are dedicating significant efforts in defining network protection directly in the optical layers.

The increase in WDM complexity brought the need for suitable control and management strategies into foreground. Problems such as routing and resource allocation for optical connections can no longer be manually solved in complex network architectures, as it usually happened in the earlier experimental WDM installations. Automatic planning tools and procedures are needed for the future which can achieve an efficient utilization of network resources in a reasonable computational time.

In the work we are presenting we would like to focus on the issue of planning and optimization of WDM networks providing failure-protected connections. The design tool we have developed is able to perform the automatic planning of the working and protection lightpaths while optimizing the amount of fibers that must be installed to satisfy all the connection requests. The optimization procedure follows a heuristic approach which allows to attain good sub-optimal results with a moderate computational effort. It can be applied to various network scenarios under the assumption that the requested connections are static and known a priori. In section 2 the basic terminology for WDM networks and the protection and restoration techniques are reviewed. Section 3 introduces and explains our heuristic optimization approach, while section 4 is dedicated to the analysis of the results obtained by applying this approach to some case-study networks.

\section{Working and spare capacity reservation techniques for an optical connection}

In WDM networks traffic is carried by means of circuit-switched transport entities, optically routed on the basis of their wavelength. These basic entities are the lightpaths (elsewhere optical-channel sections), composed of sequences of WDM channels, one per crossed link, connecting a source to a destination. A WDM link corresponds usually to a multifiber cable equipped with transmission components (e.g. optical amplifiers). Fibers are unidirectional (the number used in one propagation direction is not necessarily equal to number used in the opposite direction). In the simplified model adopted in this work we will not consider any physical transmission phenomena affecting the signal quality. The nodes of the WDM mesh network are Optical Cross Connects (OXCs): they perform all-optical switching (space and sometimes wavelength switching) on the WDM transit lightpaths, without the intervention of the electronic layers. 
In addition, they behave as terminal equipment for some lightpaths, performing the add and drop functions.

The connections requested by the nodes at a given time to a WDM network all together form the virtual (alias logical) topology: each node pair may request more than one connection. Two different traffic types may be offered to a WDM network. Static traffic is a known set of permanent connection requests assigned a priori which the network must be able to satisfy. If traffic is dynamic, connection requests arrive at random instants to the nodes of the network and connections are semi-permanent (i.e. temporary with long duration). Though dynamic lightpath provisioning is becoming more and more important, in this work we wish to consider only the static situation, leaving for future development the extension to dynamic traffic conditions. Connections must be established by suitably configuring network switching resources and allocating network transmission resources.

If WDM survivability is not supported by the network, a connection is activated by setting up one single lightpath (called working lightpath) from the source to the destination node. Setting up a lightpath implies the selection of the sequence of physical links crossed by the lightpath (routing) coupled with the selection of the wavelength channel that must be allocated to the lightpath in each link (wavelength assignment) [2,3]. Routing and Wavelength Assignment (RWA) can be interpreted as the mapping of an arc of the virtual topology over the physical topology [4]. In the case of multifiber links RWA becomes Routing, Fiber and Wavelength Assignment (RFWA), as also a particular fiber must be selected on each link for a given lightpath.

The complexity of the RFWA function [5] greatly depends on the wavelength conversion capability of the switching nodes of the network. In the present work we will consider two types of WDM networks, according to their wavelength conversion capability [6]:

Wavelength Path (WP): no conversion capability is provided;

virtual Wavelength Path (VWP): every node is fully equipped with converters so that an incoming optical channel can always be converted on any idle output wavelength.

If the connection is protected in the WDM layer, the network must be able to provide spare resources in case of failure, according to the adopted survivability scheme. WDM protection mechanisms must be simple and easy to manage, in order to guarantee a fast intervention in case of failure, as we have already pointed out in section 1 . A very good option having these features is dedicated path protection, also called Optical Channel Section (OChS) protection [7]. Each optical circuit setup between a source and a destination node (working lightpath) is backed-up by a dedicated protection lightpath having the same 
source and destination. In case of failure, the recovery operation is initiated by the end nodes of the broken lightpath and does not require reconfiguration of transit OXCs. For the path protection to be effective the two lightpaths (w/p pair for brevity) must be setup in physical route diversity, i.e. in such a way that they do not share any physical link (link-disjoint paths).

The problem of preallocating the $\mathrm{w} / \mathrm{p}$ pair to a connection request on a mesh WDM network is quite complex, since the RFWA of the working and the protection lightpaths are coupled, due to the route-diversity constraint. Moreover, since under-utilization of network resources is the necessary drawback of protection, it is important that preallocation is solved under some optimality criterion in a way to minimize the required network capacity per protected connection [8]. There are two main techniques to find two link-disjoint paths connecting two nodes of a mesh topology. The simpler one is called two-step search, while the more complex but better performing technique is the one-step search.

To understand the difference, for sake of clarity, let us consider the mesh network of Fig. 1a, having one single channel per link (one wavelength and one fiber). We further assume the lightpath length (based on distance) to be the optimality criterion. According to the two-step search, the shortest path connecting the two source-destination node pair is found (e.g. applying the Dijkstra algorithm) and it is allocated to the working lightpath. The channels already assigned to the working path are then disabled and the shortest-path algorithm runs again to route the protection lightpath. The two step search is greedy: it obtains disjoint paths that are suboptimal and there are situations in which it fails to find a solution even if the $\mathrm{w} / \mathrm{p}$ disjoint pair actually exists. The network represented in Fig. 1a is the simplest topology in which such situation does occur [9]. Fig. 1a represents the first step of the two-step search. Fig. 1b shows that, once the shortest path has been allocated to the working lightpath between nodes $A$ and $D$, there is no chance to setup the protection path because the network is disconnected. For this particular property this has been called trap network [10]. Finally, Fig. 1c shows that a pair of disjoint paths actually exists between $A$ and $D$, but the two-step search can not find it.

The one-step search, proposed by Bhandari [9], is able to overcome the twosteps limitations has been. It is named after the fact that the two lightpaths of the w/p pair are not routed separately, but they are jointly routed by performing a suitable algorithm. Besides being able to solve trap-network topologies, it can also find better solutions than the two-step search. This is because the total length of a w/p pair composed of the first- and the second-shortest paths between two nodes is not necessarily the minimum-length pair connecting the nodes. We will illustrate this concept with an example in section 4. Bhandari introduced the one-step search for a single-fiber, single-wavelength topology. In our work we started from his algorithms and we extended it to a WDM multi- 


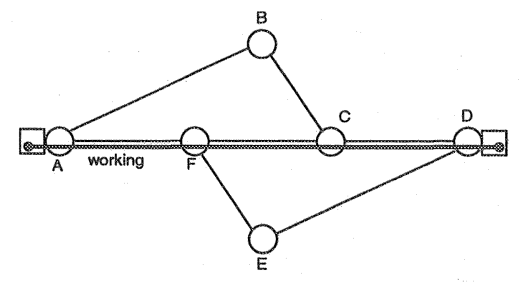

Figure 1a. Trap network: first step of the two-step algorithm.

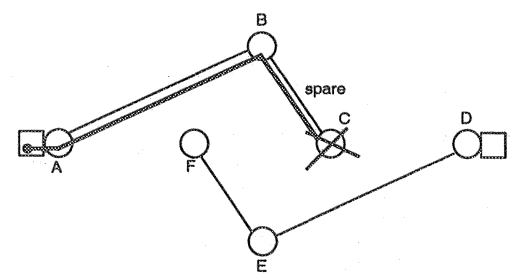

Figure $1 b$. The two-step algorithm fails to find the protection path.

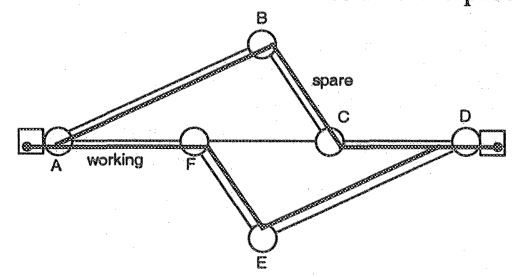

Figure 1c. The w/p disjoint paths obtained with the one-step algorithm.

fiber environment, modifying it in order to match it to the particular network model employed in our design and optimization tool.

\section{Description of the heuristic optimization method}

Static optimization of a WDM network (with or without protection) can be so summarized: given a static traffic matrix, find the optimum values of a set of network variables that minimizes a given cost function under a set of constraints. The exact solution of WDM optimization with static traffic [11], leading to the absolute minimum of the cost function, can be found by applying the Integer Linear Programming (ILP). Ref. [12] presents the results obtained by applying the ILP method on a case-study network (NFS-Net) when the linkdisjoint path-protection technique is employed. The ILP method requires quite a complex mathematical formulation and is computationally intensive when applied to large networks.

A computationally simpler alternative to ILP is the following deterministic heuristic approach, first proposed to our knowledge by the authors of Refs. $[13,5]$. The static traffic matrix is considered as a set of connections that can be setup sequentially, starting from an idle network. Lightpaths (or w/p pairs in case of path-protection) demanded according to the static traffic matrix are routed in sequence, performing RFWA upon each lightpath (or w/p pair) of the set. After all the lightpaths have been setup, the network is globally optimized by trying to tear down and reroute lightpaths according to an optimization heuristic criteria. The entire process can be then iterated. We set up a design 
tool quite similar to those presented in the references mentioned above, but with some peculiar features that make it very flexible and applicable to many network environments. In our tool all the fibers in the network host the same number of wavelengths $W$, chosen a priori. The number of fibers of each link is not a constraint in the optimization operation: links are given by the tool as many fibers as needed to route all the lightpaths requested, given $W$. In particular, each link initially contains a number of fibers so great to be considered infinite; during the optimization the amount is gradually reduced. Connections may be unprotected or protected. We assume a full protected network using a dedicated path-protection scheme. Each connection request is then satisfied by statically allocating resources for the working and the protection lightpaths which must be path-disjointed. WP, VWP and partially-VWP networks can be modeled.

After an initial sorting according to a particular rule [14], each connection request is processed. An unprotected connection is processed by setting up a lightpath by performing RFWA in an optimal "greedy" way, i.e. so that the "cost" of the resources allocated to it is the lowest possible without disrupting already setup lightpaths. The choice of the "cost" or better of the optimality criterion corresponds to the selection of a set of particular criteria for routing, fiber and wavelength assignment. The heuristic approach we followed is able to support plenty of the classical RFWA criteria, that is: "Shortest Path" (SP) and "Least Loaded Routing" (LLR) for routing, "Pack", "Spread" and "FirstFit" for fiber and wavelength assignment [14]. In particular, more criteria can be combined in a prioritized list. This means that whenever two alternative possible RWFA solutions for a lightpath result equal according to a certain criterion, the choice is made on the basis of the criterion having the next lower priority. The list of criteria to be used with their respective priority is specified by the user at the beginning of the optimization process. Another option regards SP routing: the metrics used for path measurement can be chosen between "Minimum Hop" ( $\mathrm{mH}$ ) and "Minimum Length" ( $\mathrm{mL})$, referring to the number of crossed physical links and the physical length of the path, respectively. In other similar optimization techniques presented so far in literature $[12,15]$ routing is performed in a constrained mode, considering only a pre-determined subset of paths among each node pair; on the opposite, our approach allow us to implement unconstrained routing.

All the RFWA criteria mentioned above can be modeled by adopting the layered graph (elsewhere called wavelength graph) as a working auxiliary representation of the network state. This representation, introduced by Refs. [2], has been often used for dynamic traffic analysis [2] or for static optimization in monofiber networks [16]. In Ref. [14] we have extended the use of the layered graph to multifiber network (multifiber layered graph, MLG). A powerful advantage of the MLG representation lies in the possibility to do a joint resolution of the routing, fiber and wavelength assignment problems. After the arcs of the 
MLG are assigned suitable weights, the lightpath is setup by finding the lowestweight path in the MLG. The MLG weight system allows to support multiple prioritized RFWA criteria and to model a wide range of network environments (for instance, the VWP and WP network scenarios).

Let us now explain how a path-protected connection is managed. Our model can be employed to setup the w/p pair either with a two-step or with a onestep path-disjoint search. The concept of optimality based on path length we employed in section 2 in the simple mono-fiber and mono-chromatic example is extended to comprise the choice of the fiber and the wavelength. This is easily accomplished in our model by exploiting weight-assignment of the MLG. In the two-step case RFWA is performed by setting up in the MLG first the working and then on the protection lightpath, as they were two different unprotected lightpaths. During the second step the network model must be suitably modified to keep into account the link-disjoint constraint. To perform the one-step search we have defined a new special algorithm extending the use Bhandari algorithm on a MLG. An explanation of this algorithm would require quite a lengthy description that we omit here for brevity. The computational complexity of the one-step search on the MLG is similar to the two-step.

Let us now complete the description of the operation of the design tool when dedicated path-protection is requested for all the connections. The operation is visualized by the flow-chart of Fig. 2. After the initial w/p pairs setup has been completed (starting from the idle network), all the empty fibers are pruned. Then the optimization iteration begins. An optimization counter $k$ is defined and initially set to 1 . Any fiber containing only $k$ occupied and $W-k$ unused wavelengths (let us name it a $k$-fiber) is detected. The lightpaths routed on that $k$-fiber are considered sequentially. Each of them belongs to a w/p pair: this w/p pair is tentatively deallocated and allocated again with the general RFWA criteria and path-disjoint search chosen for the optimization, this time avoiding the detected fiber. If all the $w / p$ pairs can be rerouted without meeting blocking conditions, the tentative reallocations are confirmed; otherwise the old lightpaths are maintained. When rerouting of all the lightpaths on the selected fiber has been attempted, the procedure is repeated on all the other $k$-fibers of the network. Then all the empty fibers are pruned again, $k$ is incremented and another iteration begins. The whole is repeated until $k=W$.

\section{Heuristic method application}

In the following of this paper we would like to present and comment some of the results we obtained by applying our optimization method to some particular network topologies. In each optimization experiment we monitored the following design variables upon which we measured the performance of our tool: 


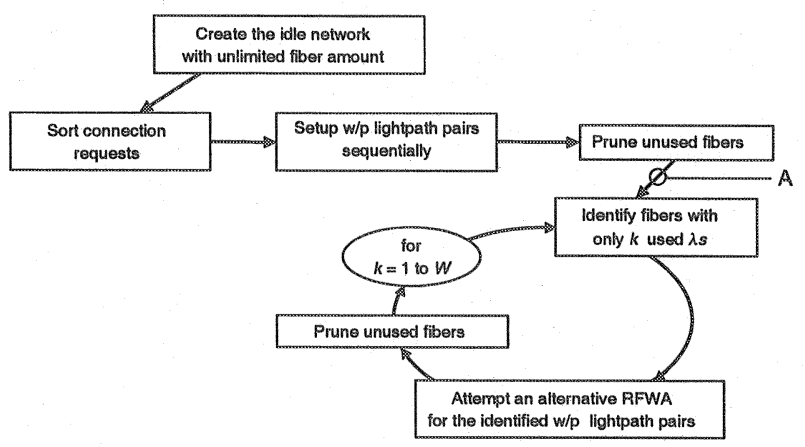

Figure 2. Flow-chart of the optimization procedure with dedicated path-protection.

- total fiber number $(M)$ : is the total number of fibers which have at least one active wavelength channel and can not therefore be pruned from the network;

- total fiber length $(L)$ : is the total physical length of the transmission resources of the network corresponding to $M$;

assigned channels $(A)$ : is the total number of wavelength-channels of the network which have been assigned to some lightpath and are therefore in use.

A lower bound of these variables can be predetermined in several ways. We have decided to consider as lower bound the so called capacity bound [7]: though it is not the tightest possible, it is quite easy to evaluate. It is obtained by assigning to each connection the w/p link-disjoint path pair, routed using the SP criterion, neglecting any wavelength conflict and assuming infinite capacity on each link. When the SP metric is $\mathrm{mH}$, then the capacity bound $\bar{A}$ of the number of assigned channels $A$ is obtained. The corresponding bound of the total fiber number is $\bar{M}=[\bar{A} / W\rceil$. Our model requires that, before performing network optimization, some decisions must be taken regarding design parameters such as initial sorting rule of the connections and RFWA algorithm. Given the heuristic nature of the optimization process, it is not easy to predict the influence of such parameters on the final result: an effective choice can be made only by directly observing the results. One of the aims of this last section is to display the effects of the variation of some design parameters: those which will not be explicitly mentioned in the discussion have already been selected in order to give the best result. Moreover, whenever SP is employed for routing, the metric used to measure path lengths is $\mathrm{mH}$ or $\mathrm{mL}$ when the cost function to be minimized is the total fiber number or the total fiber length, respectively. 


\subsection{Effects of the link-disjoint-path algorithm}

As we explained in section 3, one of the features of our design approach is the ability of performing link-disjoint routing of $\mathrm{w} / \mathrm{p}$ pairs in the one-step mode. The advantages given by this mode compared to the two-step mode strictly depend on the physical topology of the network and on the traffic matrix. In particular, the two-step mode fails to find a solution or finds sub-optimal solutions when trapnetworks are present in the link pattern connecting the source to the destination node [9]. In a well shaped network with almost balanced traffic the probability of the existence of trap-nets is quite low. In order to ascertain the advantages of the one-step algorithm applied to a WDM network we performed the optimization of an ad-hoc designed topology which presents a certain number of trap-nets. This 8-node topology, represented in Fig. 3, was originally proposed for a one-fiber and one-wavelength environment [9]. We extend its use to a multifiber and WDM environment, in the two cases with (VWP) and without (WP) wavelength converters. The traffic matrix is chosen in order to emphasize the effects of the trap-nets: a single connection is requested between all the node pairs, except for the pairs $(A, Z),(A, D)$ and $(B, Z)$, which demand 10,5 and 10 connections, respectively. This is because the majority of trap-nets can be found in the pattern connecting those nodes. To further emphasize trap-nets, physical lengths are also assigned to the links as shown in the figure.

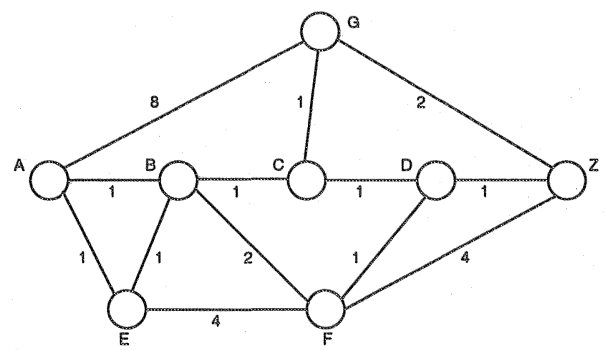

Figure 3. 8-node WDM network to study link-disjoint search algorithms. Links are labeled with their length.

Fig. 4 a represents the results in terms of total fiber length $L$ (arbitrary units) obtained by optimizing the network in the most favorable conditions, i.e. VWP and employing the one-step disjoint-path search (the prioritized set of RFWA criteria is SP-F-C-LLR, that will be defined shortly below in section 4.2). In Fig. $4 \mathrm{~b}$ we display the relative percent increment in the optimized cost parameter $\Delta_{\mathrm{x}}$, defined as: $\Delta_{\mathrm{x}}=100 \cdot\left(L_{\mathrm{x}}-L_{\mathrm{VWP}, 1}\right) / L_{\mathrm{VWP}, 1}$, where $L_{\mathrm{VWP}, 1}$ is the best total fiber length of Fig. $4 \mathrm{a}$ and $L_{\mathrm{x}}$ is the total fiber length resulting from the optimization performed in the conditions $\mathrm{x} . \mathrm{x}$ stands for (VWP,2), (WP,1) and (WP,2) when the VWP scenario is optimized using the two-step search and the WP scenario is optimized using the one- and two-step search, respectively. The 
figure shows that the effect of the choice of the link-disjoint search algorithm can be relevant for some networks and, as expected, increases with $W$. It is noticeable that the choice of the two-step search in VWP scenario can even nullify the advantages of wavelength conversion, as it happens for $W=2$ and $W=4$.

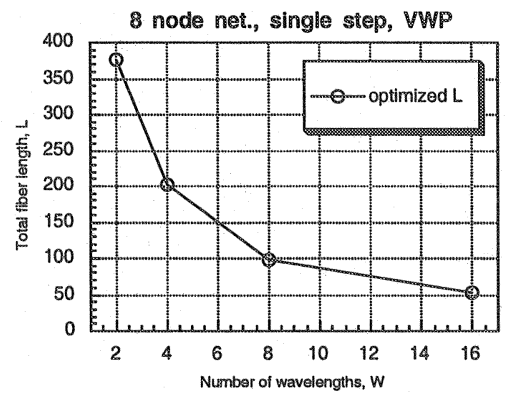

Figure 4a. Best case results obtained by the one-step link-disjoint search, (VWP case).

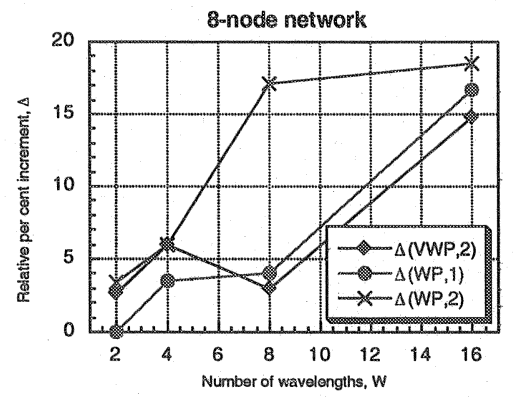

Figure $4 b$. Percent deviations $\Delta$ from the best case.

\subsection{NSF-Net optimization: effects of the RFWA criteria}

We chose to apply our design method to a well known case-study network, which is the NSF-Net (National Science Foundation Network), having 14 nodes and 22 links (Fig. 5).

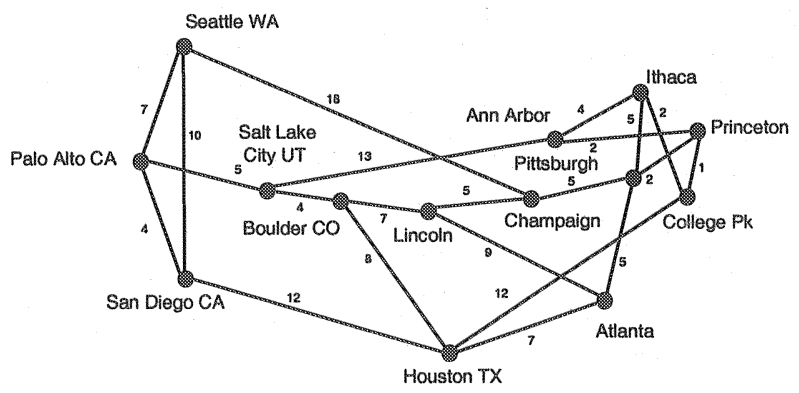

Figure 5. NSF-Net physical topology. Links are labelled with their length.

The main purpose of this set of experiments is to validate our heuristic method, comparing the results to those produced by an ILP optimization and reported in Ref. [12]. For this reason we choose the same normalized lengths (shown in Fig. 5) for the links of the physical topology and the same set of connection requests as those reported by Miyao and Saito. The data in their paper regarded the cases with $W=(2,4,8)$ and the VWP network scenario, 
having the total fiber length as cost function: accordingly, we adopted $\mathrm{mL}-\mathrm{SP}$ as routing criterion. In making the comparison (Fig. 6a), we took into account that our fibers are unidirectional and not bidirectional and our cost function does not comprise the switching equipment. The maximum difference from heuristic and ILP is found for the case with $W=2$ : the total fiber length given by heuristic is about $5 \%$ larger than the ILP result. The gap between heuristic and ILP tends to decrease when $W$ increases. The running time of our tool (running on a Pentium II PC at $450 \mathrm{MHz}$ ) is similar to the time reported in Ref. [12] (few hours with $W=32$ ). However, memory occupation is quite low (120 Mbyte), due to the efficient dynamic data structure implementing the MLG. It is well known that memory occupation is instead a serious issue in ILP techniques due to the huge number of variables and constraints.

Besides the comparison with ILP, we employed the NSF-Net with the same offered traffic to perform further experiments. We considered various cases by combining the following options: a) VWP and WP network scenarios; $b$ ) number of wavelengths $W$ belonging to the set: $(2,4,8,16,32)$. Moreover we found practically no difference in the NSF-Net when the optimization is performed employing the one- and the two-step link-disjoint search. As mentioned above, our proposed optimization method is based on the minimization of the total required fibers. To observe the difference between the greedy (point $\mathrm{A}$ in Fig. 2) solution and the optimized one, pruned by the partially used fibers, let us introduce another performance parameter, that is the percent unused capacity, defined as: $U=100 \cdot[1-A /(W \times M)]$. It measures the percent of the total number of wavelength channels $(W \times M)$ that remains idle after the setup of all the working and protection lightpaths. Fig. $6 \mathrm{~b}$ represents the percent unused capacity evaluated in a VWP scenario in two situations: before and after the optimization. Since SP is employed with full wavelength conversion, in the first situation the total amount of allocated wavelength channels is equal to the capacity bound; however there are several partially-used fibers. Fig. $6 \mathrm{~b}$ highlights the effectiveness of the heuristic optimization procedure in eliminating partially unused fibers by rerouting the lightpaths.

Fig. 7a compares the residual percent unused capacity after the optimization in the VWP and WP network scenarios when link-disjoint path protection is employed. The comparison shows that the flexibility added to the network by the wavelength converters allows to better manage the network resources. Finally, in Fig. $7 \mathrm{~b}$ we compare the case in which dedicated link-disjoint pathprotection is adopted to the case in which connections are not protected [14], in the VWP scenario. In both cases the optimization is carried out by the same heuristic procedure and with the same set of connection requests. The results indicate that when dedicated path-protection lightpaths are required the optimization procedure is more effective than in the unprotected case. 


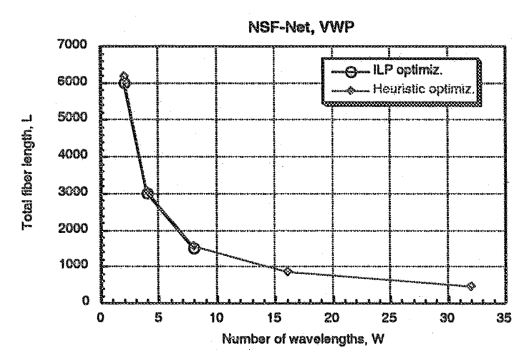

Figure $6 a$. Total fiber length $L$ obtained by our heuristic algorithm (SP-F-C-LLR criteria) compared to ILP results reported in the reference cited in the text.

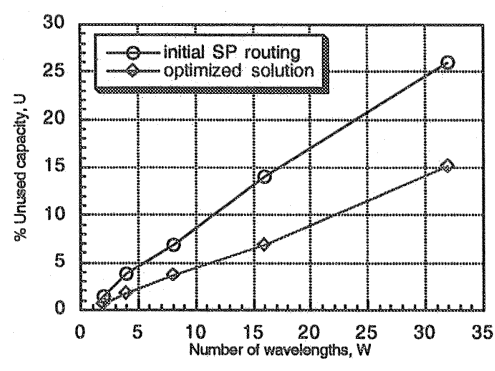

Figure $6 b$. Unused capacity before and after the optimization (VWP network scenario and path-protected connections).

The results reported above are obtained with SP-F-C-LLR. We are going to show now that SP-F-C-LLR is in the set of RFWA criteria the best one. The two routing criteria SP and LLR are combined together with the former having a higher priority and the order of letters in the sequence indicates the priorities. $\mathrm{F}$ and $\mathrm{C}$ stands for "First-Fit" applied to fiber and color (wavelength) selection, respectively. LLR probably plays a beneficial role in lowering the blocking probability during the optimization iterations, when $\mathrm{w} / \mathrm{p}$ pairs are tentatively deallocated and reallocated.

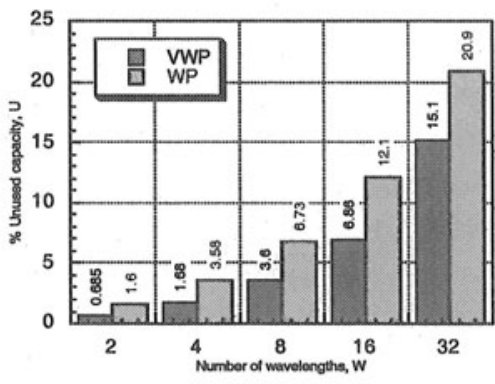

(a)

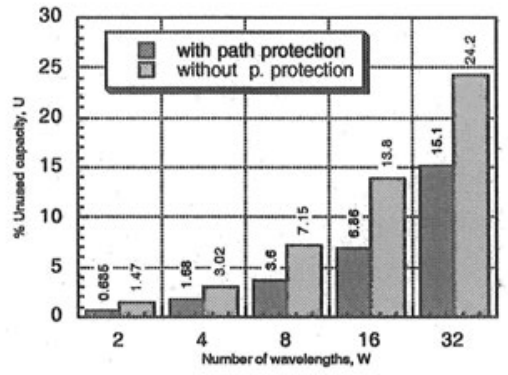

(b)

Figure 7. Unused capacity: (a) after the optimization in the WP and VWP network scenarios with link-disjoint path-protection; (b) after the optimization with and without link-disjoint pathprotection in the VWP case.

Figs. 8a and $8 \mathrm{~b}$ represent the percent relative deviations from SP-F-C-CLR of other RFWA criteria combinations in the WP and VWP network scenarios with link-disjoint path protection. When $\mathrm{F}$ and/or $\mathrm{C}$ are omitted in the combination labels it means that "Random" criterion has been used instead of "First-Fit". SPF-C-LLR is the best combination one only on average: actually for some values of $W$ (especially $W=2$ and $W=4$ ) it is overcome by other combinations. The 
main purpose of the two figures is to show how the choice of RFWA criteria is much more important when the network is not equipped with wavelength converters than when wavelength conversion is implemented. Finally we should mention that other RFWA combinations lead to a very poor optimization: e.g. if LLR is employed as unique routing criterion (without SP), $M$ is about $26 \%$ higher than SP-F-C-LLR with $W=2$ in the VWP case (data not represented in Figs. $8 \mathrm{a}$ and $8 \mathrm{~b}$ ). This is because w/p pairs are initially routed along paths longer than those selected with SP; the improvement in blocking probability given by LLR in the optimization iterations is not sufficient to compensate the initial penalty. Poor results are also obtained if priority is given to fiber or wavelength assignment criteria (e.g. with C-SP $M$ is about $11 \%$ higher than the best RFWA in the VWP case).

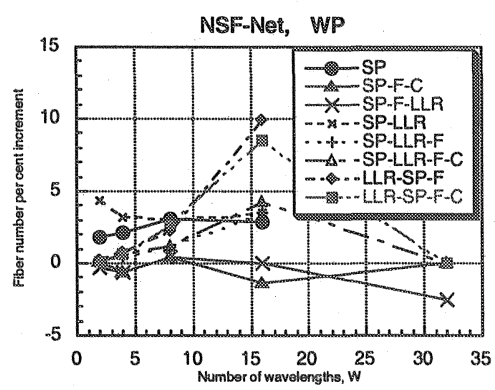

Figure $8 a$. Percent deviation of different RFWA criteria from SP-F-C-LLR, WP case.

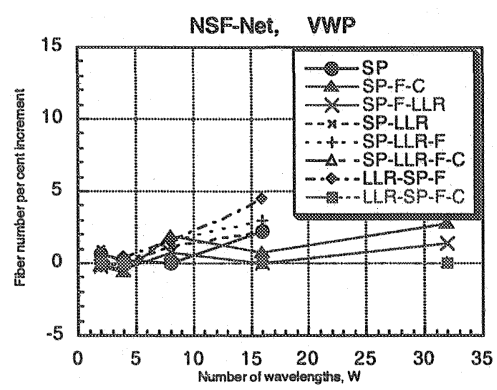

Figure $8 b . \quad$ Percent deviation of different RFWA criteria from SP-F-C-LLR, WP case.

\section{Conclusions}

In this work we have proposed a heuristic design method to optimize a resilient WDM network in a static traffic environment providing dedicated pathprotection for all the optical connection requests. The optimization procedure minimizes the total fiber length or the total fiber number. The presented design tool determines also the number of fibers of each link and solves the RFWA problem to allocate a working and a protection lightpath for each connection, guaranteeing link- (or node-)disjointness by the exploitation of two alternative path-search algorithms. When trap-nets are present in the network physical topology, we have observed that the one-step search gives better results. We applied the one-step search to a WDM multifiber environment, extending its previous usage, thanks to the MLG representation. Exploiting this graph we are able to solve physical diversity routing, fiber and wavelength assignment in an integrated way. The results presented, mainly obtained using the NSF-Net 
as case-study topology, show that our heuristic method allows to achieve good suboptimal solutions, close to the absolute ILP optimum.

\section{References}

[1] "Architectures for optical transport networks," Tech. Rep., ITU-T G872, 1999.

[2] 1. Chlamtac, A. Farago, and T. Zhang, "Lightpath (wavelength) routing in large WDM networks," IEEE Journal on Selected Areas in Communications, vol. 14, no. 5, pp. 909-913, June 1996.

[3] R. Ramaswami and K.N. Sivarajan, "Design of logical topologies for wavelength-routed optical networks," IEEE Journal on Selected Areas in Communications, vol. 14, no. 4, pp. 840-851, June 1996.

[4] B. Mukherjee, Optical communication networks, McGraw-Hill, 1997.

[5] K.-I. Sato, Advances in Transport Network Technologies - Photonic Networks, ATM, and SDH, Artech House, Norwood, MA, first edition, 1996.

[6] K.-I. Sato, "Photonic transport network OAM technologies," IEEE Communications Magazine, vol. 34, no. 12, pp. 86-94, Dec. 1996.

[7] S. Baroni, Routing and Wavelength Allocation in WDM Optical Networks, Ph.D. thesis, Department of Electronic and Electrical Engineeing, UCL London, 1998.

[8] J. Späth and H. Wei $\beta$ schuh, "Investigation of Protection Strategies: Problem Complexity and Specifics Aspects for WDM Networks," in Proceedings, NOC '99, Apr. 1999, pp. 68-75.

[9] R. Bhandari, Survivable networks, algorithms for diverse routing, Kluwer Academic Publishers, 1999.

[10] D. A. Dunn, W. D. Grover, and M. H. Gregor, "Comparison of k-shortest paths and maximum flow routing for network facility restoration," IEEE Journal on Selected Areas in Communications, pp. 88-89, 1994.

[11] N. Nagatsu, S. Okamoto, and K. I. Sato, "Optical path cross-connect system scale evaluation path accomodation design for restricted wavelength multiplexing," IEEE Journal on Selected Areas in Communications, vol. 14, no. 5, pp. 893-902, June 1996.

[12] Y. Miyao and H. Saito, "Optimal Design and Evaluation of Survivable WDM Transport Networks," IEEE Journal on Selected Areas in Communications, vol. 16, no. 7, pp. 1190-1198, Sept. 1998.

[13] N. Nagatsu, Y. Hamazumi, and K.-I. Sato, "Optical path accomodation designs applicable to large scale networks," IEICE Transactions on Communications, vol. E78-B, no. 4, pp. 597-607, Apr. 1995.

[14] G. Maier, A. Pattavina, L. Roberti, and T. Chich, "Static-lightpath design by heuristic methods in multifiber WDM networks," in Proceedings, Opticomm 2000 SPIE Conf., Oct. 2000 , pp. 64-75.

[15] S. Baroni, R.J. Gibbens P. Bayvel, and S.K. Korotky, "Analysis and Design of Resilient Multifiber Wavelength-Routed Optical transport networks," IEEE Journal of Lightwave Technology, vol. 17, pp. 743-758, may 1999.

[16] C. Chen and S. Banerjee, "A new model for optimal routing and wavelength assignment in wavelength division multiplexed optical networks," in Proceedings, IEEE INFOCOM, 1996, vol. 2a.4.1-8, pp. 164-171. 